\title{
Control system for reducing weld spatter during the welding process by using high-speed visual sensing
}

\author{
Mai SUGAWARA*, Kaoru KIYOMITSU**, Rui TAKANO**, Izumi FUJIWARA**, Yu YAMAUCHI***, \\ Takayoshi NAKAYAMA ${ }^{* * *}$ and Norimichi TSUMURA** \\ * Faculty of Education, University of Teacher Education Fukuoka \\ 1-1 Akamabunkyo-machi, Munakata, Fukuoka 811-4192, Japan \\ ** Department of Information Processing and Computer Sciences, Graduate School of Advanced Integration Science, Chiba University \\ 1-33 Yayoi-cho Inage-ku Chiba-shi, Chiba 263-8522, Japan \\ E-mail: tsumura@faculty.chiba-u.jp \\ *** Nishihara Electronic Ltd. \\ 6-8-30 Nishihara Kashiwa-shi, Chiba 277-0885, Japan
}

Received: 15 December 2016; Revised: 17 April 2017; Accepted: 1 October 2017

\begin{abstract}
Robotic welding has been the biggest use in many industry. It is important to control weld robots without weld spatter for small size target. To prevent flying weld spatter, the precursory phenomenon of weld spatter must be detected, and power source of robotic welding must be controlled to reduce before weld spatter occurs. This study aimed to propose a control system to prevent the occurrence of flying weld spatter during laser or gas tungsten arc (GTA) welding process in real-time. Because the strategy involves detecting the precursory phenomenon at high-speed, we simplified the recognition system as much as possible. The system consists of a lighting system to illuminate the weld pool, a high-speed camera and an FPGA board. The control algorithm of this system used image data information for a histogram. We analyzed the images from the high-speed camera during welding. Our analysis found that, just before the weld spatter is generated, very bright pixels temporarily and abnormally increased as the precursory phenomenon. According to the proposed system, it is possible to prevent the occurrence of weld spattering in real time during the welding process. This integration of visual feedback in a robotic welding system enhances the quality of the weld work.
\end{abstract}

Keywords : Robotic welding, Weld spatter, Robot sensing systems, High-speed sensing, Feedback, Control

\section{Introduction}

Robotic welding has become the prevailing form of welding in many industries (Pires, et al., 2006, Kah, et al., 2015). Traditionally, the welding process has been intended for large-sized workpieces in the electronics, automotive, and aircraft industries. Recently, implementations of robotic laser welding systems have been increasing in many other industries, including the manufacture of small medical instruments and commodity goods such as the titanium frames of eyeglasses. It is important to control weld robots without weld spatter, particularly for small-sized targets. Weld spatter is droplets of molten material generated at the weld pool during the welding process. Weld spatter increases costs because it needs to be cleaned up, wastes material, and causes poor weld quality, such as the lack of welding by material loss.

The subjects of many discussions of quality monitoring are monitoring the welding state and detecting the weld spatter that had occurred (Kah, et al., 2015, Daihatsu Motor Co., Ltd., 1999, Schwab, et al., 2008, Jager and Hamprecht, 2009, Schwab, et al., 2009, Olsson, et al., 2009, Eriksson, et al., 2010, Nishihara Electronic Ltd., 2011).

However, in previous works, post-welding analyses of weld spatter were generally conducted. Therefore, a new welding control is in demand to prevent the occurrence of weld spattering in real-time before it happens. To prevent flying weld spatter, the precursory phenomenon of weld spatter must be detected, and power source of robotic welding must be controlled to reduce before weld spatter occurs. No such systems exist yet. 
In this paper, we propose a control system to prevent the occurrence of flying weld spatter during laser or gas tungsten arc (GTA) welding process in real-time. The strategy involves detecting the precursory phenomenon at high-speed. Image sensing is not a bottleneck for achieving high performance because the speed is adequate, but performing the calculation processing is a bottleneck. By performing the calculation processing at high-speed, we can control the system. Based on this limitation, we simplify the recognition system as much as possible.

\section{System configuration}

The control system consists of a lighting system such as a halogen lamp over optical fiber to illuminate the weld pool, a high-speed camera that is located symmetrically to the lighting system in the vertical direction of the center of the weld pool, and an FPGA board, as shown in Fig. 1. The angle to the horizontal plane formed by the lighting output and the high-speed camera is preferably $60 \sim 70^{\circ}$. Fig. 2 shows an overhead view of the settings. As shown in Figs. 1 and 2 , the relation of the lighting system and the high-speed camera is symmetrical with respect to the target. Because the illumination light emitted from the output to the target reflects the weld pool made during welding is illuminated into the high-speed camera, and the image has a certain degree of brightness.

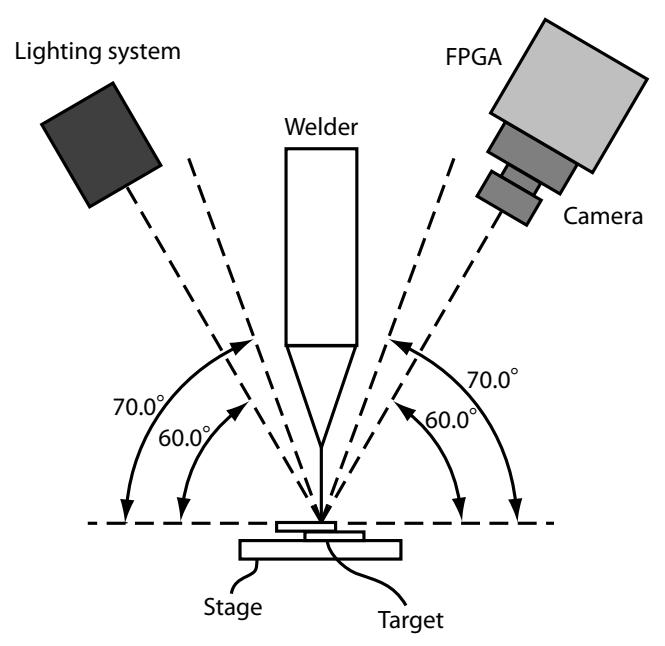

Fig. 1 System settings

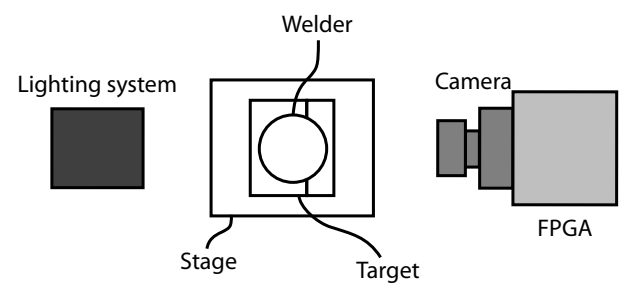

Fig. 2 Overhead view of the settings

\subsection{Lighting system}

We use a halogen lamp with an output peak in the near-infrared wavelength band (730 $800 \mathrm{~nm})$. This halogen lamp still have a power at the wavelength of $962.5 \mathrm{~nm}$ which is the filter used on this system. By using the halogen lamp in the near-infrared wavelength band, the weld pool is appropriately irradiated, and thus it is possible to acquire a functional image. The halogen lamp is placed in a position separated from the target, and the light is guided to the vicinity of the target through optical fiber and irradiated to the target. In this setup, the position of the lamp can be set at an arbitrary position.

\subsection{High-speed camera}

A lens and a filter are mounted on the high-speed camera. The filter can transmit light at the wavelength of $962.5 \mathrm{~nm}$. By providing the filter to block the light emitted by arc discharge, information from the weld pool is obtained accurately. 
As the image sensor, two pairs of CL-600 (nac Image Technology Inc., image size: $244 \times 168$, frame rate: $10000 \mathrm{fps}$ ) are arranged to obtain an image of $320 \times 240$ pixels. Fig. 3 shows an image at the time of normal welding. In this experiment, the welding process is GTA welding, the welding current is $70 \mathrm{~A}$, and the target is a lap welding of aluminum. As shown in Fig. 3, in the case of GTA welding, the aluminum target is melted by the discharge from the torch and generates a formed weld pool. The weld pool is visually recognized at almost the center as $1 / 6$ of the size of the image frame (about $1 / 2$ of the horizontal size and $1 / 3$ of the vertical size).

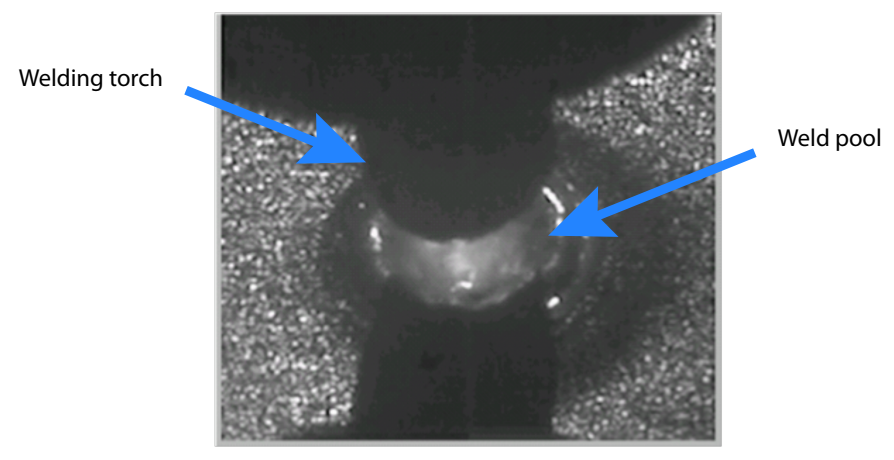

Fig. 3 Image at the time of normal welding

The data signal output from the high-speed camera is input into the FPGA evaluation board for image processing. However, the FPGA evaluation board cannot be directly connected to the data signal output from the high-speed camera due to the electrical properties of the FPGA. For this reason, we designed and created a level conversion board to match the order of the electrical level. Fig. 4 shows a system block diagram. Fig. 5 shows a photograph of the connection.

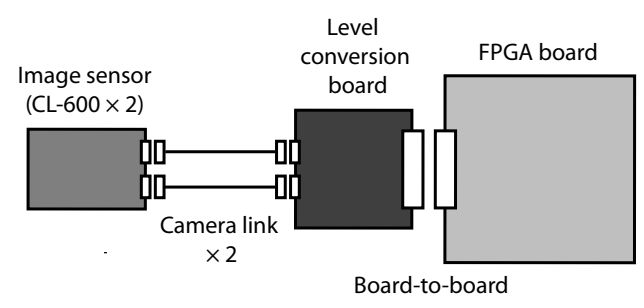

Fig. 4 System block diagram

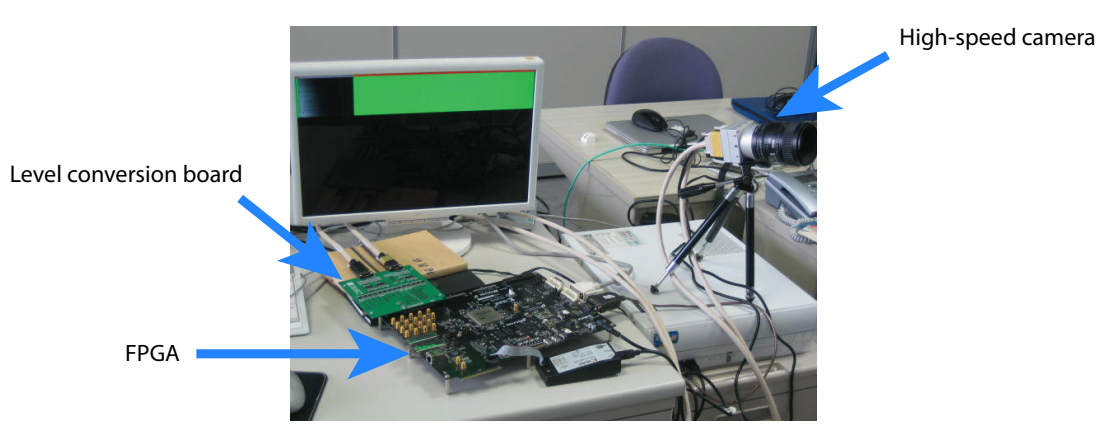

Fig. 5 Photograph of the connection

\subsection{FPGA}

For the image processing board, calculation processing is commonly performed with a conventional CPU. However, when using the CPU, input images might be lost, or processing to the output might be delayed. Therefore, by using an FPGA: Xilinx Virtex-6, these problems can be solved. The FPGA can perform real-time processing in the hardware, and it is not like the CPU, which consumes the internal logic when it performs complicated calculations. Performing the calculation in the FPGA enables real-time processing of the images.

The FPGA board monitors the pixel count at each gray level of the image data for one frame in real time, and it outputs a trigger signal when the precursory phenomenon of the weld spatter is detected. Controlled reduction of the 
power supply to the welder by a trigger signal can prevent the occurrence of weld spatter. Fig. 6 shows a photograph of the actual device.

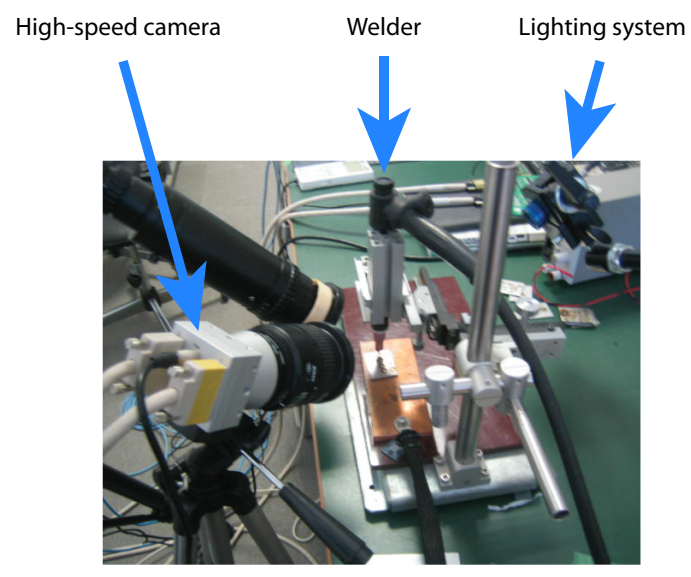

Fig. 6 Photograph of the actual device

\section{Control algorithm}

A block diagram of the internal FPGA is shown in Fig. 7. First, image data are received from the high-speed camera. Second, the pixel count of each gray level in one flame is calculated. Last, determination of the precursory phenomenon of weld spatter is made in the decision block. The algorithm was implemented in FPGA with hardware language.

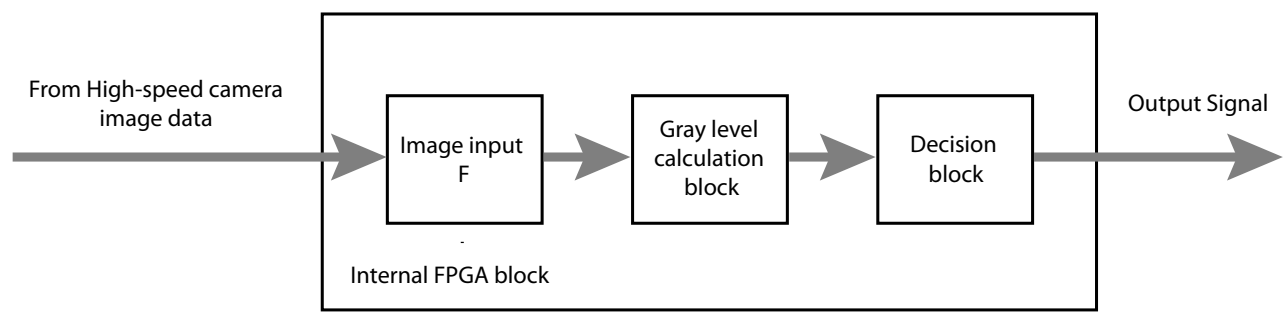

Fig. 7 Internal FPGA

\subsection{Generation of the histogram}

In the image processing module, we use the image information for a histogram. A histogram is calculated at each frame of the image. Fig. 8 shows a histogram of the image data at the time of normal welding. In this graph, the horizontal axis represents the gray-level values of the image data, and the vertical axis represents the number of pixels. The range of the bead at the time of welding is expanded vertically and horizontally to be about one-third of the screen. The brightness is adjusted so that the gray level value of the peak pixel count of the histogram becomes about $40 \sim 50$ in all 256 shades during normal welding. The setting condition of the high-speed camera is shown in Table 1 . The frame rate is $6200 \mathrm{fps}$. In the image, pixel counts of the gray level between $40 \sim 70$ are frequently observed, and the peak of the pixel counts is also observed between the $40 \sim 70$. The pixel counts where pixel values are higher than 70 decrease with increasing pixel value, and so the pixel count is zero at the highest pixel values in the image.

Table 1 Setting conditions of the high-speed camera

\begin{tabular}{l|c}
\hline Setting items & Values \\
\hline Image size & $320 \times 240$ \\
Frame rate & $6200 \mathrm{fps}$ \\
\hline
\end{tabular}

However, from the analysis of the video of the welding in the experiment, we found that, just before the weld spatter is generated, a small new peak appears in the histogram where the pixel values are between the 200 and 255 levels, and 


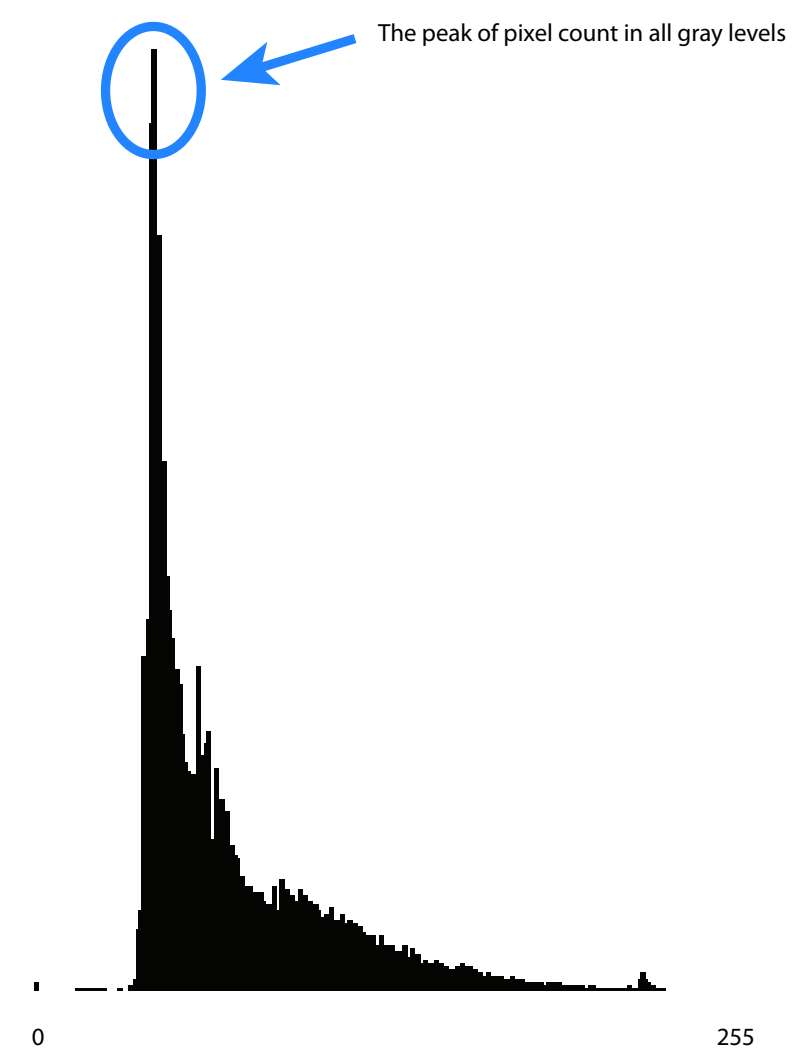

Fig. 8 Histogram at the time of normal welding

where the pixel counts are small in the ordinal condition. In particular, first, we identify the flame at the time of the flying weld spatter particle shown in Fig. 9 by image processing based on optical flow (Barron, et al., 1994) and fast Fourier transform (Potts, et al., 2001), and by skilled human. Next, the characteristics of the precursory phenomenon in the previous frame of the identified frame of the spatter are analyzed. Fig. 10 shows the histogram of the image data at the time of abnormal welding, just before the weld spatter is generated.

In other words, just before the weld spatter is generated, very bright pixels of the $200 \sim 255$ levels are seen. That bright pixels observed continuously until the weld spatter is generated; the phenomenon will leave lasting and the duration time of the phenomenon can be regarded as long enough. These temporarily and abnormally increase at the time of the precursory phenomenon due to irregular reflection and interference by the disturbance of the surface state of the weld pool. When the FPGA board detects the precursory phenomenon of weld spatter, by outputting a trigger signal, it is possible to force suppression control. The detailed and exact cause of the high intensity spots by the disturbance of the surface state is still unclear, but several theories exist; for example, a luminous body might occur with high luminance from the weld pool, such as a laser-induced plume or plasma, and gas bubbles (Chen, et al., 1996, Kang and Na, 2005, Schweier, et al., 2013, Heider, et al. 2013). In any case, sensing the high-intensity spots is closely related to detecting signs of flying spatter.

In this proposed system, the frame rate is $6200 \mathrm{fps}$. The frame rate is not limited to this particular rate, but it is preferable to use a frame rate that is greater than $5000 \mathrm{fps}$. In this research, to detect the spatter with more than $200 \mu \mathrm{s}$ duration time, minimum frame rate should be $5000 \mathrm{fps}$. Therefore, in this experiment we use the camera with $6200 \mathrm{fps}$. The high-brightness luminous phenomenon can be observed variously as is shown in Table 2. Table 2 shows the duration time between the beginning of high-brightness luminous phenomenon and spatter with $20000 \mathrm{fps}$. Our system can detect 12 spatters in 18 spatters in $5000 \mathrm{fps}$ imaging. Since we have measured the welding process by $20000 \mathrm{fps}$ imaging, we can emulate the imaging process with lower fps than $20000 \mathrm{fps}$ by flame thinning process. In the evaluation of the system, we supposed that the computational power is strong enough. Image quality is not measured in this experiment. It is noted that the above result that tell $6200 \mathrm{fps}$ imaging is suitable for our system is obtained by changing the sampling rate in the computer. 

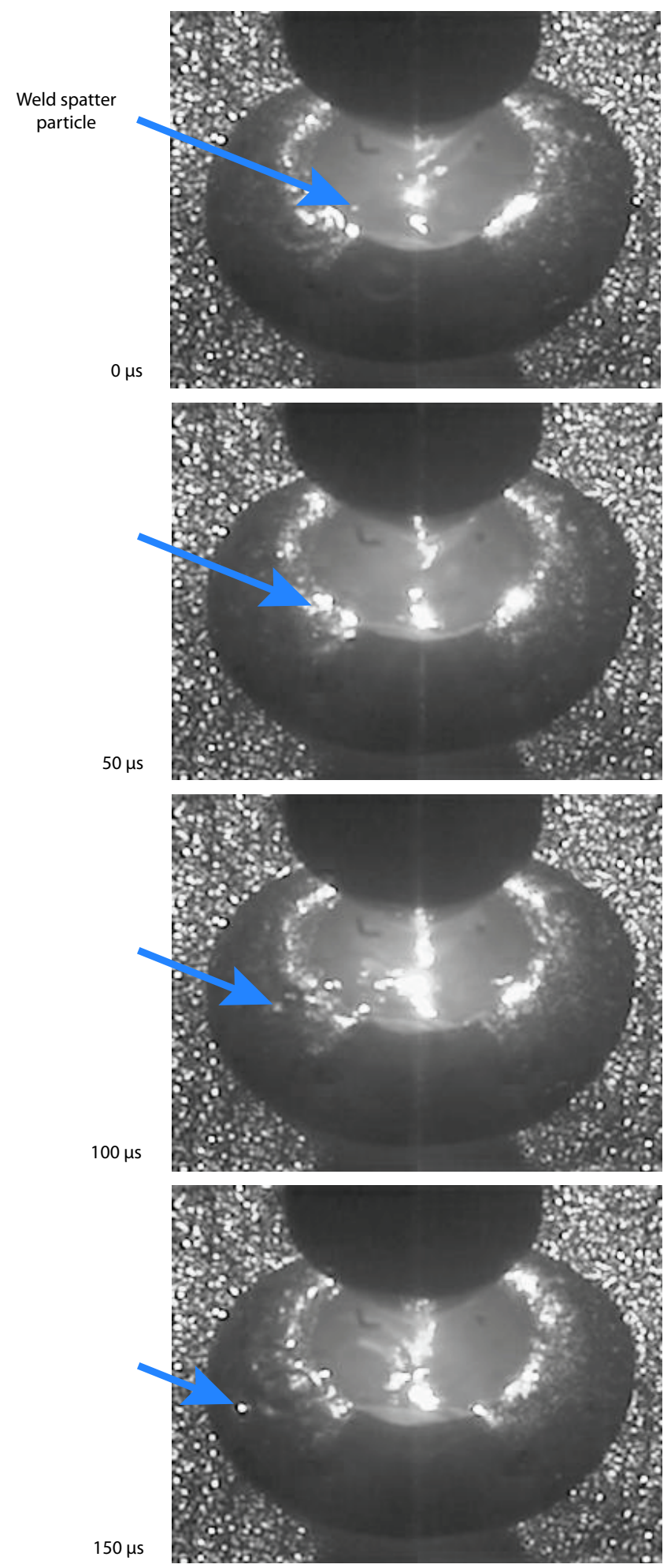

Fig. 9 Images at the time of a flying weld spatter particle

\subsection{Determination of precursory phenomenon by thresholds}

According to the results from a case analysis, we found two types of thresholds. The weld spattering phenomenon is highly likely to appear, when the sum of pixel counts $I_{j}$ of each high gray levels more than 200 defined as belows is increased to 150 or more when acquiring an image having a total number of pixels per frame as 76800 .

- Pixel counts of each gray levels: $\left\{I_{j} \mid j \in \mathbb{N}, 200 \leq j \leq 255\right\}$ 


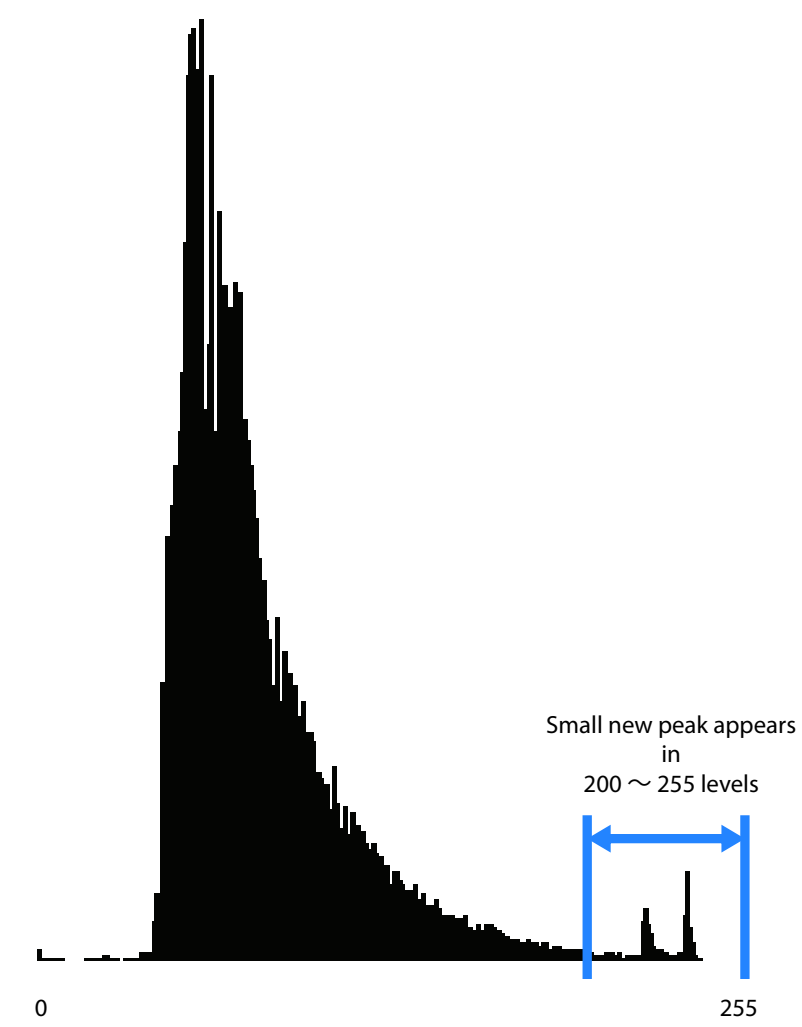

Fig. 10 Histogram for an easy-to-understand example at the time of abnormal welding, just before the weld spatter is generated

In addition, the weld spattering phenomenon is extremely likely to appear, when a specific pixel number $I_{j}$ is increased to 10 or more when acquiring an image having a total number of pixels per frame as 76800 . In the case that the weld spattering phenomenon does not occur, the pixel number for each brightness level over 200 is about 1 or 2 counts/level. On the one hand, we found 10 counts/level, just before the spattering phenomenon.

Therefore, for such a high gray level, an increase in the number of pixels is considered a sign of the weld spattering phenomenon, and so the feedback system should be sent a signal $S$ for controlled reduction of the power supply to the welding machine. This is defined in the following equation,

$$
S= \begin{cases} & \left(\text { if } \quad \sum I_{j} \geq T_{1}\right) \\ & \left(\text { if } \quad \max \left(I_{j}\right) \geq T_{2}\right) \\ 0 & \text { (otherwise) }\end{cases}
$$

\section{$T_{1}$ and $T_{2}$ are thresholds.}

The thresholds are given as follows.

- $T_{1}=150$

- $T_{2}=10$

If the total sum of the pixel counts for brightnesses level over 200 is greater than 150 , inclusive-OR, the pixel count in any one level of those levels is greater than 10, then the FPGA board outputs a trigger signal.

Because the frame rate in the experiment is $6200 \mathrm{fps}$, by processing the image acquired from the high-sensitivity camera at an extremely high speed, it can realize an image processing method of detecting an abnormal change in the image by using the FPGA board in real time. As shown in Table 2, our system on the detection method and the thresholds can detect with $66 \%$ success rate. The method to control the laser or GTA welding apparatus to prevent weld spatter can 
Sugawara, Kiyomitsu, Takano, Fujiwara, Yamauchi, Nakayama and Tsumura,

Mechanical Engineering Letters, Vol.3 (2017)

Table 2 Observed high-brightness luminous phenomenon

\begin{tabular}{l|r|r|r|r}
\hline Data sets & \multicolumn{2}{|c|}{ Frame No. of occurrences event } & Frame distance of event & Duration time $[\mu$ s $]$ \\
\cline { 2 - 3 } & Spatter & Precursory phenomenon & & - \\
\hline Data A & 2358 & - & - & 23800 \\
& 3151 & 2675 & 476 & 200 \\
& 5282 & 5278 & 4 & 17150 \\
& 5621 & 5278 & 343 & 1750 \\
& 6496 & 6461 & 35 & - \\
\hline Data B & 8032 & - & - & 22700 \\
& 1396 & 942 & 454 & 10700 \\
& 1683 & 1469 & 214 & 900 \\
& 3497 & 3479 & 18 & 9050 \\
& 3690 & 3509 & 181 & 4500 \\
& 4234 & 4144 & 90 & 1500 \\
& 5245 & 5215 & 30 & 11350 \\
& 6381 & 6154 & 227 & - \\
& 9244 & - & - & 15900 \\
& 10726 & 10408 & 318 & - \\
& 10897 & - & - & - \\
& 11050 & - & - & - \\
\hline
\end{tabular}

be realized based on the trigger signal $S$ from image processing that can detect signs of the weld spattering phenomenon by adjusting the power supply of the weld torch, or by controlling the supply nozzle of the filler wire.

\section{Conclusions}

In this paper, we presented a control system that detects the precursory phenomenon of weld spatter, with the aim to prevent the occurrence of weld spatter by image processing during the welding process. Robotic welding is widely used in many industries. Moreover, implementations of robotic laser welding systems have recently been increasing in many industries, including the manufacture of small medical instruments and commodity goods such as the titanium frames of eyeglasses. It is important to control weld robots without weld spatter for small-sized targets. However, to date, a welding control that can prevent the occurrence of weld spattering before happens, is not been previously developed. To prevent flying weld spatter, the precursory phenomenon of weld spatter should be detected, and the power source of robotic welding should be controlled to reduce before the occurrence of weld spatter. No such systems exist yet.

We developed a control system for laser or gas tungsten arc (GTA) welding processwa in real time for small-sized targets by using high-speed visual sensing. The system consists of a lighting system such as a halogen lamp over optical fiber to illuminate the weld pool, a high-speed camera set with to the vertical direction of the center of the weld pool and symmetrically located to the lighting system, and an FPGA board.

The control algorithm of this system uses image data information for a histogram. We analyzed the images from the high-speed camera during welding. From this analysis, we found that, just before the weld spatter is generated, very bright pixels temporarily and abnormally increased as the precursory phenomenon. We found that as the pixel counts of high brightness levels increased, the weld spattering phenomenon was highly likely to occur. A method to control the laser or GTA welding apparatus without weld spatter can thus be realized based on the trigger signal from the image processing to detect the sign of the weld spattering phenomenon by adjusting the power supply of the weld torch or controlling the supply nozzle of the filler wire (typically, increasing the wire feed speed promotes heat radiation).

This sensing can be widely applied to the weld-monitoring system. Such integration of visual feedback in a robotic welding system can improve the quality of the weld work. According to the proposed system, it is possible to prevent the occurrence of weld spattering in real time before the spattering occurs during the welding process. This method has the advantage of reducing the industrial waste caused by weld spattering. Moreover, it can increase the in-service rate up by reducing the number of nozzle cleanings and spatter removals. In future work, the proposed method will be evaluated by using the system in an actual application. 


\section{References}

Barron, J. L., Fleet, D. J. and Beauchemin, S. S., Performance of optical flow techniques, International Journal of Computer Vision, Vol. 12, No. 1 (1994), pp. 43-77.

Chen, J. H., Sun, Z. C. and Fan, S. D., Study on the mechanisms of spatter produced by basic welding electrodes, Welding Journal, Vol. 75, No. 10 (1996), pp.311-316.

Daihatsu Motor Co., Ltd., Weld spattering amount evaluation method (in Japanese) (Patent style), Japanese Unexamined Patent Application Publication No. Hei 11-347728, December 21 (1999).

Eriksson, I., Powell, J. and Kaplan, A. F. H., Signal overlap in the monitoring of laser welding, Measurement Science and Technology, Vol. 21, No. 10 (2010), 105705 (7pp).

Heider, A., Sollinger, J., Abt, F., Boley, M., Weber, R. and Graf, T., High-speed X-ray analysis of spatter formation in laser welding of copper, Physics Procedia, Vol. 41 (2013), pp. 112-118.

Jager, M. and Hamprecht, F. A., Principal component imagery for the quality monitoring of dynamic laser welding processes, IEEE Transactions on Industrial Electronics, Vol. 56, No. 4 (2009), pp. 1307-1313.

Kah, P., Shrestha, M., Hiltunen, E. and Martikainen, J., Robotic arc welding sensors and programming in industrial applications, International Journal of Mechanical and Materials Engineering, Vol. 10, No. 13 (2015), pp.1-16 (online).

Kang, S.K. and Na, S.J., A mechanism of spatter production from the viewpoint of the integral of specific current action, Welding Journal, vol. 84 (2005), pp. 188s-196s.

Nishihara Electronic Ltd., Defective electrode detecting device (Patent style), WIPO Patent Application WO/2011/162417, December 29 (2011).

Olsson, R., Eriksson, I., Powell, J. and Kaplan, A., Pulsed laser weld quality monitoring by the statistical analysis of reflected light, Proceedings of the 5th International WLT-Conference on Lasers in Manufacturing (2009), pp. 369374.

Pires, J. N., Loureiro, A. and Bolmsjö, G., Welding robots: Technology, system issues and applications, London: Springer-Verlag (2006).

Potts, D., Steidl, G. and Tasche, M., Fast Fourier transforms for nonequispaced data: A tutorial, In Modern sampling theory (pp. 247-270). Birkhäuser Boston (2001).

Schwab, G., Vincent, T. L. and Steele, J. P. H., Contaminant classification in robotic gas metal arc welding via image based spatter tracking, Proceedings of the 17th IEEE International Conference on Control Applications (2008), pp. 751-756.

Schwab, G., Steele, J. P. H. and Vincent, T. L., Vision-Based spatter classification for contaminant detection, Welding Journal, Vol. 88 (2009), pp.121s-130s.

Schweier, M., Heins, J.F., Haubold, M.W. and Zaeh, M.F., Spatter formation in laser welding with beam oscillation, Physics Procedia, Vol. 41 (2013), pp. 20-30. 